\title{
Description de la nymphe de Telopelopia fascigera (Verneaux) (Diptera, Chironomidae)
}

\author{
H. Laville ${ }^{1}$ \\ M. Viaud-Chauvet 1
}

Une description détaillé de la nymphe de Telopelopia fascigera (Verneaux), inconnue jusqu'à ce jour, permet de préciser les caractères qui en font un genre plus apomorphe que le genre Conchapelopia. $T$. fascigera parait être une espece eurytherme adaptée au potamon des grandes rivières.

Deacription of the pupa of Telopelopia fascigera (Verneaux) (Diptera, Chironomidae).

A detailed description of the pupa of Telopelopia foscigera (Verneaux), unknown until now, has allowed the precise description of characters that make the genus more apomorphic than the genus Conchapelopia. $T$. fascigera appears to be a eurythemic species adapted to the potamon of large rivers.

\section{1. - Introduction}

Le genre Telopelopia fut créé par Roback (1971) pour une espèce de Tanypodinae néarctique, Telopelopia okoboji (Walley), primitivement decrite par Walley (1928, sub Tanypus okoboji).

Verneaux (1970) décrit l'imago ơ d'une nouvelle espèce Conchapelopia fascigera que Murray (1980) classa plus tard dans le genre Telopelopia defini par Roback, établissant ainsi la répartition holarctique du genre.

Dans ce même travail, Murray décrit un deuxième représentant paléarctique récolté au Maroc : Telopelopia maroccana Murray retrouvé depuis dans les Balkans (Fittkau et Reiss 1978).

Les stades jeunes - larve et nymphe - des représentants de ce genre étaient inconnus jusqu'à ce jour.

Des dérives au fil de l'eau effectuées en juin 1981 dans le Potamon du Tam, rivière issue de la zone méridionale du Massif Central et affluent rive droite de la Garonne, contenaient $1 \mathrm{~N} \circ$ et 47 exuvies nymphales de Telopelopia fascigera ${ }^{2}$. C'est ce maté-

1. Laboratoire d'Hydrobiologie, ERA 702 du C.N.R.S., Univer. site Paul Sabatier, 118, route de Narbonne, 31062 Toulouse Cedex (France).

2. Holotype nymphal et paratypes : Collection Laville, Toulouse (France) ; autres paratypes Zoologische Staatssammlung, Münich (R.F.A.). riel qui nous permet de décrire la première nymphe appartenant à ce genre et de préciser sa position systématique par rapport au genre voisin Conchapelopia.

\section{2. - Description de la nymphe}

Longueur : $5,4-5,9 \mathrm{~mm}$ chez la $९(\mathrm{n}=5) ; 4,8$ $5,5 \mathrm{~mm}$ chez le o $(\mathrm{n}=5)$.

Coloration : exuvie brun clair avec une zone légèrement plus foncée au bord médio-oral des tergites II à VIII. Bords antérieurs des tergites et sternites II à VIII avec une ligne brun foncé (apophyses). Tergite $I$ avec 2 lignes médianes parallèles plus colorées.

- Come thoracique (fig. 1 a) grosse, aplatie, aussi longue (ou légèrement moins longue) que le premier segment abdominal; environ 2 fois et demi plus longue que large, progressivement élargie depuis la base jusqu'à son extrémité distale arrondie. Sa forme rappelle celle de Conchapelopia sp. Fulda (Fittkau 1962 : fig. 169).

Crible de moitié aussi large que le plus grand diamètre de la corne, de dimension inférieure à ceux de la plupart des Conchapelopia. De forme ovale, il est entouré par une auréole plus ou moins ovoïde dont le diamètre atteint le double de celui du crible.

La chambre de la corne garnit toute la lumière de celle-ci et présente deux lobes antérieurs proéminents qui encadrent le crible. La membrane de la 


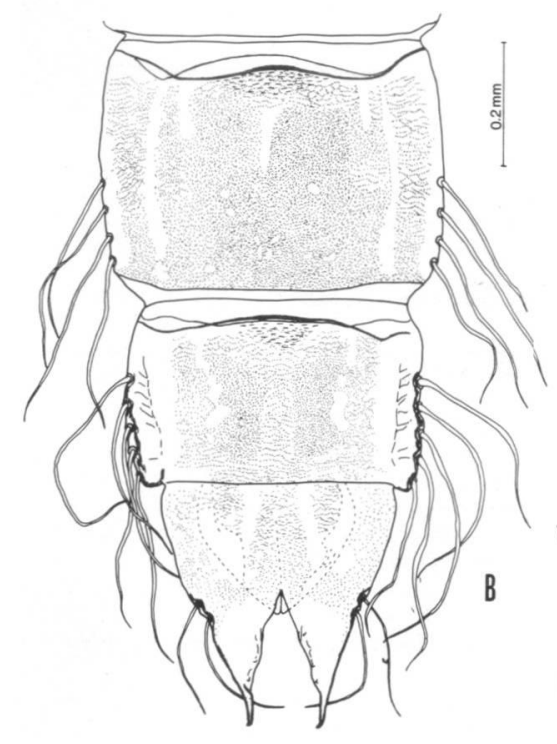

Fig. 1. Nymphe $\sigma$ de Telopelopia fascigera (Verneaux): A : Corne thoracique : B : Tergites abdominaux VII-IX;

C : Extrémité du segment anal (vue dorsale).

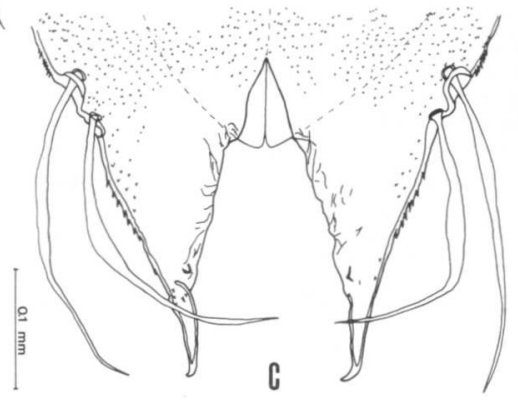

corne est structurée en écailles terminées par de fines spinules dans leur partie distale. Bord du stigmate aplati, au moins 4 fois plus long que haut.

- Thorax : bord frontal, entre les cornes, garni d'un chagrin de très fines spinules dorsales devenant progressivement plus fortes et émoussées sur la face dorsale où se différencie une rangée de (7-10) dents hautes de 12 à $18 \mu$, allant de la base de la corne vers la région antérieure. Ces dents sont dirigées du cen. tre vers le bord latéral du thorax : cette rangée de dents peut être assimilée à un " peigne thoracique ". Les autres parties du thorax sont lisses et les tuber-

cules thoraciques absents. Soies mésonotales (Mth) inégales ( 20 à $105 \mu$ ), Mth2 atteint 1/4 de Mth1 et 1/5 de Mth3. Mthl est éloignée de 2 fois sa longueur de Mth2 et de 3 fois sa longueur de Mth3. Mth2 est éloignée de Mth3 de la méme distance. Mth2 est située au-dessous de Mthl et est plus proche de celle-ci que de Mth3. Mth3 est également éloignée de Mth1 et de Mth2.

- Abdomen (fig. 1 b). Segments abdominaux garnis d'un dense chagrin de fines spinules fourchues surtout dans la partie médioanale du tergite comme chez Conchapelopia pallidula (Mg) (Fittkau 1962 : fig. 
180 à 182). Dans la zone médiane et orale des tergites, les spinules sont plus courtes, plus espacées et alignées jusqu'à former des rangées transversales au voisinage du bord oral. Le chagrin atteint le bord anal jusqu'à la membrane de l'intersegment.

Soies dorsales (D) et ventrales (V) tubuleuses et hyalines, longues environ de 30 à $50 \mu$.

4 soies $D$ sur les tergites II et VIII ; 5 soies D sur les tergites III à VI. Soies L (soies latérales des segments I - VI) un peu plus longues $(60 \mu)$ que les soies D. 2 soies $\mathrm{L} 1$ et $\mathbf{L} 2$ de chaque côté des tergites $I$ à VI, 1 soie L2 ventrale dans l'angle du sternite VII.

Les soies LS (soies latérales tubuleuses) s'insèrent sur la moitié anale du segment VII et sur un peu moins des $2 / 3$ anaux du segment VIII.

Le segment VII a 4 soies LS de la longueur du segment $(400-430 \mu)$; le segment VIII a 5 soies LS plus longues $(470-500 \mu)$, d'environ 1 fois et demi la longueur du segment.

Le segment anal (IX) (fig. 1 b et 1 c), légèrement plus long que large avec un chagrin dans la moitié orale : bord externe droit ou légèrement concave garni de fines spinules; bord interne légèrement convexe, sans spinules avec une très petite dent située vers le $1 / 3$ distal de la pointe du lobe anal. Extrémité du lobe anal effilée et recourbée ventra. lement à son apex. Les étuis gonopodiaux dépassent légèrement la moitié des lobes anaux.

Les 2 soies LS de chaque lobe sont presque aussi longues que le segment anal ; la soie antérieure est insérée au centre du lobe.

\section{3. - Remarques taxonomiques}

Dans la lignée Thienemannimyia de la Tribu des Pentaneurini définie par Fittkau (1962) et sur la base des caractères morphologiques de l'imago $\sigma$ de Telopelopia fascigera, Murray (1980) conclut à une plus grande affinité du genre Telopelopia pour Conchapelopia que pour Arctopelopia.

Les caractères morphologiques de la nymphe précédemment définis confirment cette affinité avec le genre Conchapelopia. Telopelopia fascigera parait ètre une forme plus évoluée comme plusieurs caractères apomorphiques de la nymphe le montrent, notamment :

- la réduction de la taille du crible de la corne et la disparition de la chambre de la corne en forme de tube plus ou moins régulier ;

- l'acquisition d'un "peigne thoracique " bien différencié ;

- la réduction de la longueur des soies abdominales :

- l'allongement et l'effilement des pointes du segment anal.

Tous ces caractères permettent de différencier la nymphe de Telopelopia fascigera des nymphes du genre Conchapelopia.

\section{4. - Ecologie et répartition}

Telopelopia fascigera est une espèce seulement connue de France. Verneaux (1970) la signale pour la première fois dans trois cours d'eau du Centre et de l'Est : le Doubs inférieur (2 stations), la Saône (2 stations) et la Seine (1 station à Montereau).

Nos récoltes proviennent toutes de trois stations de la zone aval de la rivière Tarn, à Reynies (alt. $90 \mathrm{~m}$ ), Montauban $(85 \mathrm{~m})$ et La Française $(74 \mathrm{~m})$. Dans cette zone, la rivière est très large (115-130 m), sa pente très faible $(0,03 \%)$; l'amplitude des températures est de $18^{\circ} \mathrm{C}$ (minima $5.6^{\circ} \mathrm{C}$; maxima $23-24^{\circ} \mathrm{C}$ ).

L'analyse des données chimiques de ces trois stations situées à l'aval de trois agglomérations d'inégale importance indique un niveau moyen de pollution. Entre les agglomérations de Montauban et de la Française, le Tarn reçoit la rivière Aveyron où $T$. fascigera n'a jamais été récoltée.

Cette espèce paraît donc bien limnophile et eurytherme ; elle peut caractériser le potamon des grandes rivières de plaine et des fleuves. Selon Verneaux (1970), les cours d'eau où elle a été récoltée présentent une forte amplitude thermique annuelle et un degré élevé de trophie. Les récoltes sur le Tarn, situées à l'aval des rejets polluants de trois agglomérations, corroborent les observations de cet auteur.

Les récoltes imaginales de Verneaux (1970) se situent entre mi-juillet et début septembre. Sur le Tarn nos récoltes de Nymphe ou d'Exuvies nymphales sont toutes du 30 juin 1981. Les stations de la Seine à Montereau au S.E de Paris, et celles du Tarn inférieur appartiennent à la zone 13 de la Limnofauna Europaea. 
Dans les trois stations du Tarn : $T$. fascigera a été récoltée en compagnie des trois Tanypodinae : Ablabesmyia longistyla, Rheopelopia ormata et Nilotanypus dubius.

\section{Travaux cités}

Fittkau (E.J.). 1962. - Die Tanypodinae (Diptera, Chironomidae). Abh. Larvalsyst. Insekten Berlin : $453 \mathrm{p}$.

Fittkau (E.J.) et Reiss (F.). 1978. - Chironomidae. In Illies J., ed. Limnofaura Europaea, G. Fischer, Stuttgart : 404440.
Murray (D.A.). 1980. - Telopelopia maroccana sp. n. a second palearctic species of Telopelopia Roback (Diptera, Chironomi dae). Acta Universitatis Carolinae. Biologica, 1978: $151-156$.

Roback(S.S.). 1971. - The Adults of the Sub Family Tanypodinae (= Pelopiinae) in North America (Diptera, Chironomidae). Monogr. Acad. nat. Sci. Phila, 17:410 p.

Verneaux (J.). 1970. - Conchapelopia fascigera $\mathrm{n}$. sp, : une nouvelle espece de Tanypodinae (Diptera - Chironomidae). Description l'imago $\sigma$ et données écologiques. Ann. sci. Univ. Besancon. Zool. $6: 59.64$.

Walley (G.S.). 1928. - The Genus Tanypus in Canada, with a key to the North American Species. Anm ent. Soc. Amer. 21: 581-593. 